\title{
A Dice Rolling Game on a Set of Tori
}

\author{
Jeehoon Kang \\ Department of Computer Science and Engineering, \\ Seoul National University, \\ Seoul 151-742, Korea.
}

\author{
Suh-Ryung Kim* \\ Department of Mathematics Education, \\ Seoul National University, \\ Seoul 151-742, Korea.
}

\author{
Boram Park $^{\dagger \dagger}$ \\ DIMACS, \\ Rutgers University, \\ Piscataway, NJ 08854, United States.
}

Submitted: Mar 3, 2011; Accepted: Feb 29, 2012; Published: Mar 9, 2012

Mathematics Subject Classification: 15A24, 97A90

\begin{abstract}
Some linear algebraic and combinatorial problems are widely studied in connection with $\sigma$-games. One particular issue is to characterize whether or not a given vector lies in the submodule generated by the rows of a given matrix over a commutative ring. In general, one can solve this problem easily and algorithmically using the linear algebra over commutative ring. However, if the matrix has some combinatorial structure, one may expect that some more can be asserted instead of merely giving an algorithm. A recent outstanding example appeared in this line of research is the paper by Florence and Meunier published in Journal of Algebraic Combinatorics in 2010. In the same spirit, we consider a matrix over $\mathbb{Z}_{n}$ to completely characterize the submodule generated by its rows and give a constructive proof. The main idea for the characterization is to find certain good basic elements in the row space and then express a given element as the linear combination of them as well as some additional term.
\end{abstract}

Keywords $\sigma$-game, Fiver, dice rolling game on a torus, dice rolling game on a circle, system of linear equations

*This research was supported by the National Research Foundation of Korea(NRF) grant funded by the Korea government(MEST) (No. 2010-0009933).

${ }^{\dagger}$ This work was supported by National Research Foundation of Korea Grant funded by the Korean Government, the Ministry of Education, Science and Technology (NRF-2011-357-C00004).

$\ddagger$ Corresponding author: boramp@dimacs.rutgers.edu 


\section{Introduction}

We introduce a dice rolling game on a set of tori as a variant of Fiver. Fiver is a puzzle game in which you need to flip over all the counters so that your $n \times n$ board changes from being completely full of white pieces, and, instead, becomes entirely inhabited by black pieces. Clicking on any counter will not only flip that piece from being white to black (or vice versa), but its 4 neighboring pieces immediately above, below, to the left and right of the piece that you clicked will also reverse their allegiance, becoming black if they were white, or white if they were black. The game is named after Fiver the rabbit from the classic book and movie, Watership Down.

Fiver is a $\sigma$-game and was studied by Hunziker et al. [4] and Lee and Yang [5]. See [1$3,6,7]$ for some results on $\sigma$-games. Some linear algebraic and combinatorial problems are widely studied in connection with $\sigma$-games. One particular issue is to characterize whether or not a given vector lies in the submodule generated by the rows of a given matrix over a commutative ring. In general, one can solve this problem easily and algorithmically using the linear algebra over commutative ring. However, if the matrix has some combinatorial structure, one may expect that some more can be asserted instead of merely giving an algorithm. A recent outstanding example appeared in this line of research is the paper by Florence and Meunier [2]. In the same spirit, this paper considers a matrix over $\mathbb{Z}_{n}$ corresponding to a rectangular array on a torus and completely characterizes the submodule generated by its rows.

Given a positive integer $n$, an $n$-dice is a dice with $n$ faces such that element $i$ of $\mathbb{Z}_{n}$ is written on the $i$ th face. Given positive integers $\alpha_{1}, \alpha_{2}$, we arbitrarily locate $\alpha_{1} \alpha_{2} n$-dice in an $\alpha_{1} \times \alpha_{2}$ rectangular array, and glue the lower and upper together and also the left and right edges. Then we have $\alpha_{1} \alpha_{2} n$-dice on a torus (see Figure 1 ).

We denote by $\mathcal{D}\left(\left(\alpha_{1}, \alpha_{2}\right), n\right)$ the set of tori on each of which $\alpha_{1} \alpha_{2} n$-dice are located as described above. For positive integers $\beta_{1}, \beta_{2}, \beta_{1} \leq \alpha_{1}, \beta_{2} \leq \alpha_{2}$, we call the following action a "( $\left.\beta_{1}, \beta_{2}\right)$-rolling procedure" (see Figure 1 ).

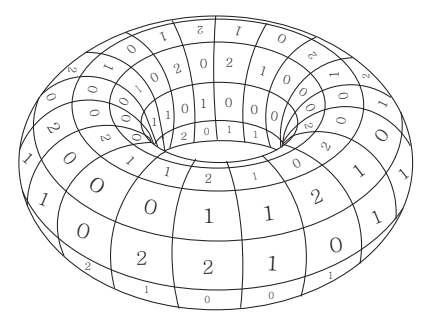

Figure 1: A torus belonging to $\mathcal{D}((19,8), 3)$

We roll the $n$-dice which form a $\beta_{1} \times \beta_{2}$ rectangular array on the torus so that we increase the number on the top face of each of them by 1 .

Then we may ask "Given a torus in $\mathcal{D}\left(\left(\alpha_{1}, \alpha_{2}\right), n\right)$, is it possible to have 0 appear on the top face of each of $\alpha_{1} \alpha_{2} n$-dice on the torus by repeatedly applying $\left(\beta_{1}, \beta_{2}\right)$-rolling proce- 


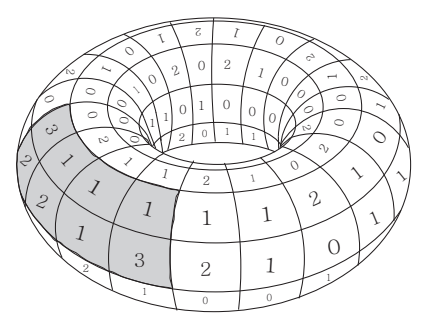

Figure 2: The torus resulting from going through a $(2,4)$-rolling procedure applied to the shaded array of the torus given in Figure 1

dures?" We call this game the dice rolling game on $\mathcal{D}\left(\left(\alpha_{1}, \alpha_{2}\right), n\right)$ with respect to $\left(\beta_{1}, \beta_{2}\right)$ rolling procedures or the $\left(\left(\alpha_{1}, \alpha_{2}\right) ;\left(\beta_{1}, \beta_{2}\right) ; n\right)$-DR game for short. We say that a torus for which the answer to the above question is yes is a solution of the $\left(\left(\alpha_{1}, \alpha_{2}\right) ;\left(\beta_{1}, \beta_{2}\right) ; n\right)-D R$ game. Given positive integers, $\alpha_{1}, \alpha_{2}, n, \beta_{1}, \beta_{2}, \beta_{1} \leq \alpha_{1}, \beta_{2} \leq \alpha_{2}$, we will characterize the tori which are solutions of the $\left(\left(\alpha_{1}, \alpha_{2}\right) ;\left(\beta_{1}, \beta_{2}\right) ; n\right)$-DR game in the rest of this paper.

We define a module over $\mathbb{Z}_{n}$ as follows. We denote the set of $\alpha_{1} \times \alpha_{2}$ matrices with elements in $\mathbb{Z}_{n}$ by $\mathcal{M}\left(\left(\alpha_{1}, \alpha_{2}\right), n\right)$. For each element $A \in \mathcal{M}\left(\left(\alpha_{1}, \alpha_{2}\right), n\right)$, we denote by $[A]_{i, j}$ the element in the $(i, j)$-entry. We define operations on $\mathcal{M}\left(\left(\alpha_{1}, \alpha_{2}\right), n\right)$ in terms of addition and multiplication over $\mathbb{Z}_{n}$ : Given two matrices $A, B \in \mathcal{M}\left(\left(\alpha_{1}, \alpha_{2}\right), n\right)$,

$$
[A+B]_{i, j}=[A]_{i, j}+[B]_{i, j} \quad \text { and } \quad[c A]_{i, j}=c[A]_{i, j}
$$

for any $c \in \mathbb{Z}_{n}, 0 \leq i \leq \alpha_{1}-1,0 \leq j \leq \alpha_{2}-1$. Throughout this paper, we assume that the 1st component and the 2nd component of every subscript are reduced to modulo $\alpha_{1}$ and modulo $\alpha_{2}$, respectively. Then we associate a torus in $\mathcal{D}\left(\left(\alpha_{1}, \alpha_{2}\right), n\right)$ with a matrix in $\mathcal{M}\left(\left(\alpha_{1}, \alpha_{2}\right), n\right)$ whose $(i, j)$-element equals the number on the top face of the $n$-dice in the $(i, j)$ position of the torus for $i, j, 0 \leq i \leq \alpha_{1}-1,0 \leq j \leq \alpha_{2}-1$. In this way, we can give an isomorphism between $\mathcal{D}\left(\left(\alpha_{1}, \alpha_{2}\right), n\right)$ and $\mathcal{M}\left(\left(\alpha_{1}, \alpha_{2}\right), n\right)$. Thus, in order to characterize the solutions of the $\left(\left(\alpha_{1}, \alpha_{2}\right) ;\left(\beta_{1}, \beta_{2}\right) ; n\right)$-DR game, it is sufficient to characterize the matrices whose corresponding tori are its solutions. Let $E_{i, j}$ denote $\alpha_{1} \times \alpha_{2}$ matrix with 1 in the $(i, j)$-entry and 0 elsewhere. We define a matrix $J_{k_{1}, k_{2}}^{m_{1} \times m_{2}}$ by

$$
J_{k_{1}, k_{2}}^{m_{1} \times m_{2}}=\sum_{i=0}^{m_{1}-1} \sum_{j=0}^{m_{2}-1} E_{k_{1}+i, k_{2}+j}
$$

for integers $m_{i}, k_{i}$ such that $1 \leq m_{i} \leq \alpha_{i}$ for $i=1,2$. For example, let $\alpha_{1}=6, \alpha_{2}=8$, $\beta_{1}=2, \beta_{2}=2$, and $n=5$. Then

$$
J_{1,2}^{2 \times 2}=\begin{array}{|c|c|c|c|c|c|c|c|}
\hline 0 & 0 & 0 & 0 & 0 & 0 & 0 & 0 \\
\hline 0 & 0 & 1 & 1 & 0 & 0 & 0 & 0 \\
\hline 0 & 0 & 1 & 1 & 0 & 0 & 0 & 0 \\
\hline 0 & 0 & 0 & 0 & 0 & 0 & 0 & 0 \\
\hline 0 & 0 & 0 & 0 & 0 & 0 & 0 & 0 \\
\hline 0 & 0 & 0 & 0 & 0 & 0 & 0 & 0 \\
\hline
\end{array}, \quad J_{5,5}^{3 \times 4}=\begin{array}{|c|c|c|c|c|c|c|c|c|}
\hline 1 & 0 & 0 & 0 & 0 & 1 & 1 & 1 \\
\hline 1 & 0 & 0 & 0 & 0 & 1 & 1 & 1 \\
\hline 0 & 0 & 0 & 0 & 0 & 0 & 0 & 0 \\
\hline 0 & 0 & 0 & 0 & 0 & 0 & 0 & 0 \\
\hline 0 & 0 & 0 & 0 & 0 & 0 & 0 & 0 \\
\hline 1 & 0 & 0 & 0 & 0 & 1 & 1 & 1 \\
\hline
\end{array} .
$$


In particular, we denote $J_{k_{1}, k_{2}}^{\beta_{1} \times \beta_{2}}$ by $J_{k_{1}, k_{2}}^{*}$. The torus corresponding to $A$ in $\mathcal{M}\left(\left(\alpha_{1}, \alpha_{2}\right), n\right)$ is a solution of the $\left(\left(\alpha_{1}, \alpha_{2}\right) ;\left(\beta_{1}, \beta_{2}\right) ; n\right)$-DR game if the zero matrix $O$ can be obtained by adding a linear combination of $J_{., \text {to }}^{*}$. That is, $A$ is a solution of the $\left(\left(\alpha_{1}, \alpha_{2}\right) ;\left(\beta_{1}, \beta_{2}\right) ; n\right)$ DR game if and only if there exist $c_{i, j} \in \mathbb{Z}$ satisfying the system of linear equations

$$
A+\sum_{j=0}^{\alpha_{2}-1} \sum_{i=0}^{\alpha_{1}-1} c_{i, j} J_{i, j}^{*}=O
$$

In this aspect, we call $A$ a solution matrix of the $\left(\left(\alpha_{1}, \alpha_{2}\right) ;\left(\beta_{1}, \beta_{2}\right) ; n\right)$-DR game. We call matrix $\left(c_{i, j}\right)$ a solving coefficient matrix of the $\left(\left(\alpha_{1}, \alpha_{2}\right) ;\left(\beta_{1}, \beta_{2}\right) ; n\right)$-DR game corresponding to $A$. We characterize the matrices in $\mathcal{M}\left(\left(\alpha_{1} \times \alpha_{2}\right), n\right)$ which are solutions of the $\left(\left(\alpha_{1}, \alpha_{2}\right) ;\left(\beta_{1}, \beta_{2}\right) ; n\right)$-DR game in the following section. The main idea is to find certain good basic elements in the row space and then express a given element as the linear combination of them as well as some additional term.

\section{Solution matrices of the $\left(\left(\alpha_{1}, \alpha_{2}\right) ;\left(\beta_{1}, \beta_{2}\right) ; n\right)$-DR game}

In this section, we characterize the solution matrices of the $\left(\left(\alpha_{1}, \alpha_{2}\right) ;\left(\beta_{1}, \beta_{2}\right) ; n\right)$-DR game. Throughout this paper, for $i=1,2$, let $g_{i}, r_{i}, s_{i}$ denote the integers such that

$$
g_{i}=\operatorname{gcd}\left(\alpha_{i}, \beta_{i}\right), \quad \alpha_{i}=r_{i} g_{i}, \quad \beta_{i}=s_{i} g_{i} .
$$

For each integer $j$, by the division algorithm, there exist integers $u_{j}, w_{j}$ satisfying

$$
j \equiv u_{j} \quad\left(\bmod g_{1}\right) \quad 0 \leq u_{j} \leq g_{1}-1, \quad j \equiv w_{j} \quad\left(\bmod g_{2}\right) \quad 0 \leq w_{j} \leq g_{2}-1 .
$$

First note that for integers $i$ and $j$,

$$
\sum_{a=0}^{\beta_{1}-1} E_{u_{a}, j}=s_{1} \sum_{a=0}^{g_{1}-1} E_{a, j} \quad \text { and } \quad \sum_{b=0}^{\beta_{2}-1} E_{i, w_{b}}=s_{2} \sum_{b=0}^{g_{2}-1} E_{i, b} .
$$

For integers $i, j, k_{1}, k_{2}$ such that $1 \leq k_{1} \leq \alpha_{1}$ and $1 \leq k_{2} \leq \alpha_{2}$, we define matrices

$$
\begin{aligned}
C_{i, j}^{k_{1} \times 1} & =J_{i, w_{j}}^{k_{1} \times 1}-J_{i, j}^{k_{1} \times 1} \\
R_{i, j}^{1 \times k_{2}} & =J_{u_{i}, j}^{1 \times k_{2}}-J_{i, j}^{1 \times k_{2}} \\
Q_{i, j} & =E_{u_{i}, w_{j}}-E_{i, w_{j}}-E_{u_{i}, j}+E_{i, j} .
\end{aligned}
$$

Note that, by definitions,

$$
\begin{array}{cl}
C_{i, j}^{k_{1} \times 1}=O & \text { if } 0 \leq j \leq g_{2}-1, \\
R_{i, j}^{1 \times k_{2}}=O & \text { if } 0 \leq i \leq g_{1}-1, \\
Q_{i, j}=O & \text { if } 0 \leq i \leq g_{1}-1 \text { and } 0 \leq j \leq g_{2}-1 .
\end{array}
$$


For example, consider the $((6,8) ;(2,2) ; 5)-D R$ game. Then $g_{1}=\operatorname{gcd}(6,2)=2$ and $g_{2}=\operatorname{gcd}(8,2)=2$. We take $i=3$ and $j=7$. Then $u_{3}=1, w_{7}=1$. Consider $C_{1,7}^{2 \times 1}$, that is, by definition,

$$
C_{1,7}^{2 \times 1}=\begin{array}{|c|c|c|c|c|c|c|c|}
\hline 0 & 0 & 0 & 0 & 0 & 0 & 0 & 0 \\
\hline 0 & 1 & 0 & 0 & 0 & 0 & 0 & -1 \\
\hline 0 & 1 & 0 & 0 & 0 & 0 & 0 & -1 \\
\hline 0 & 0 & 0 & 0 & 0 & 0 & 0 & 0 \\
\hline 0 & 0 & 0 & 0 & 0 & 0 & 0 & 0 \\
\hline 0 & 0 & 0 & 0 & 0 & 0 & 0 & 0 \\
\hline
\end{array} .
$$

By the definition of $J_{i, j}^{*}$,

$$
\begin{aligned}
& J_{1,1}^{*}-J_{1,2}^{*}=\begin{array}{c|c|c|c|c|c|c|c|c|}
\hline 0 & 0 & 0 & 0 & 0 & 0 & 0 & 0 \\
\hline 0 & 1 & 0 & -1 & 0 & 0 & 0 & 0 \\
\hline 0 & 1 & 0 & -1 & 0 & 0 & 0 & 0 \\
\hline 0 & 0 & 0 & 0 & 0 & 0 & 0 & 0 \\
\hline 0 & 0 & 0 & 0 & 0 & 0 & 0 & 0 \\
\hline 0 & 0 & 0 & 0 & 0 & 0 & 0 & 0 \\
\hline
\end{array} \\
& J_{1,3}^{*}-J_{1,4}^{*}=\begin{array}{|c|c|c|c|c|c|c|c|}
\hline 0 & 0 & 0 & 0 & 0 & 0 & 0 & 0 \\
\hline 0 & 0 & 0 & 1 & 0 & -1 & 0 & 0 \\
\hline 0 & 0 & 0 & 1 & 0 & -1 & 0 & 0 \\
\hline 0 & 0 & 0 & 0 & 0 & 0 & 0 & 0 \\
\hline 0 & 0 & 0 & 0 & 0 & 0 & 0 & 0 \\
\hline 0 & 0 & 0 & 0 & 0 & 0 & 0 & 0 \\
\hline
\end{array}, \\
& J_{1,5}^{*}-J_{1,6}^{*}=\begin{array}{|c|c|c|c|c|c|c|c|}
\hline 0 & 0 & 0 & 0 & 0 & 0 & 0 & 0 \\
\hline 0 & 0 & 0 & 0 & 0 & 1 & 0 & -1 \\
\hline 0 & 0 & 0 & 0 & 0 & 1 & 0 & -1 \\
\hline 0 & 0 & 0 & 0 & 0 & 0 & 0 & 0 \\
\hline 0 & 0 & 0 & 0 & 0 & 0 & 0 & 0 \\
\hline 0 & 0 & 0 & 0 & 0 & 0 & 0 & 0 \\
\hline
\end{array} .
\end{aligned}
$$

It can be checked that

$$
C_{1,7}^{2 \times 1}=\left(J_{1,1}^{*}-J_{1,2}^{*}\right)+\left(J_{1,3}^{*}-J_{1,4}^{*}\right)+\left(J_{1,5}^{*}-J_{1,6}^{*}\right)
$$

or

$$
C_{1,7}^{2 \times 1}+\left(J_{1,2}^{*}-J_{1,1}^{*}\right)+\left(J_{1,4}^{*}-J_{1,3}^{*}\right)+\left(J_{1,6}^{*}-J_{1,5}^{*}\right)=O .
$$

In addition, it is true that

$$
C_{2,7}^{2 \times 1}=\begin{array}{|c|c|c|c|c|c|c|c|}
\hline 0 & 0 & 0 & 0 & 0 & 0 & 0 & 0 \\
\hline 0 & 0 & 0 & 0 & 0 & 0 & 0 & 0 \\
\hline 0 & 1 & 0 & 0 & 0 & 0 & 0 & -1 \\
\hline 0 & 1 & 0 & 0 & 0 & 0 & 0 & -1 \\
\hline 0 & 0 & 0 & 0 & 0 & 0 & 0 & 0 \\
\hline 0 & 0 & 0 & 0 & 0 & 0 & 0 & 0 \\
\hline
\end{array}
$$


Therefore, $C_{1,7}^{2 \times 1}$ and $C_{2,7}^{2 \times 1}$ are solutions matrices of the $((6,8) ;(2,2) ; 5)$-DR game. In addition, the matrix $Q_{3,7}$ can be represented by using $C_{1,7}^{2 \times 1}$ and $C_{2,7}^{2 \times 1}$, as

$$
Q_{3,7}=\begin{array}{|c|c|c|c|c|c|c|c|}
\hline 0 & 0 & 0 & 0 & 0 & 0 & 0 & 0 \\
\hline 0 & 1 & 0 & 0 & 0 & 0 & 0 & -1 \\
\hline 0 & 0 & 0 & 0 & 0 & 0 & 0 & 0 \\
\hline 0 & -1 & 0 & 0 & 0 & 0 & 0 & 1 \\
\hline 0 & 0 & 0 & 0 & 0 & 0 & 0 & 0 \\
\hline 0 & 0 & 0 & 0 & 0 & 0 & 0 & 0 \\
\hline
\end{array}
$$

Since $C_{1,7}^{2 \times 1}$ and $C_{2,7}^{2 \times 1}$ are solution matrices, $Q_{3,7}$ is a solution matrix of the $((6,8) ;(2,2) ; 5)$ DR game. In fact, the following lemma holds.

Lemma 2.1. For any integers $i$ and $j$, matrices $C_{i, j}^{\beta_{1} \times 1}, R_{i, j}^{1 \times \beta_{2}}$, and $Q_{i, j}$ are solution matrices of the $\left(\left(\alpha_{1}, \alpha_{2}\right) ;\left(\beta_{1}, \beta_{2}\right) ; n\right)$-DR game.

Proof. Since each of linear congruence equations $j-u_{j} \equiv x \beta_{1}\left(\bmod \alpha_{1}\right)$ and $j-w_{j} \equiv x \beta_{2}$ $\left(\bmod \alpha_{2}\right)$ has a solution, there exist positive integers $\zeta_{j}$ and $\eta_{j}$ satisfying that

$$
j-u_{j} \equiv \zeta_{j} \beta_{1} \quad\left(\bmod \alpha_{1}\right) \quad \text { and } \quad j-w_{j} \equiv \eta_{j} \beta_{2} \quad\left(\bmod \alpha_{2}\right) .
$$

To show that $C_{i, j}^{\beta_{1} \times 1}$ are $R_{i, j}^{1 \times \beta_{2}}$ are solution matrices, it is sufficient to show

$$
\begin{aligned}
C_{i, j}^{\beta_{1} \times 1} & =\sum_{m=0}^{\eta_{j}-1}\left(J_{i, w_{j}+m \beta_{2}}^{*}-J_{i, w_{j}+m \beta_{2}+1}^{*}\right), \\
R_{i, j}^{1 \times \beta_{2}} & =\sum_{m=0}^{\zeta_{i}-1}\left(J_{u_{i}+m \beta_{1}, j}^{*}-J_{u_{i}+m \beta_{1}+1, j}^{*}\right) .
\end{aligned}
$$

From the definition given in (1.2), it immediately follows that $J_{i, j}^{*}=\sum_{b=0}^{\beta_{2}-1} J_{i, j+b}^{\beta_{1} \times 1}$. Then (2.9) holds as

$$
\begin{aligned}
\sum_{m=0}^{\eta_{j}-1}\left(J_{i, w_{j}+m \beta_{2}}^{*}-J_{i, w_{j}+m \beta_{2}+1}^{*}\right) & =\sum_{m=0}^{\eta_{j}-1}\left[\left(\sum_{b=0}^{\beta_{2}-1} J_{i, w_{j}+m \beta_{2}+b}^{\beta_{1} \times 1}\right)-\left(\sum_{b=0}^{\beta_{2}-1} J_{i, w_{j}+m \beta_{2}+b+1}^{\beta_{1} \times 1}\right)\right] \\
& =\sum_{m=0}^{\eta_{j}-1} \sum_{b=0}^{\beta_{2}-1}\left(J_{i, w_{j}+m \beta_{2}+b}^{\beta_{1} \times 1}-J_{i, w_{j}+m \beta_{2}+b+1}^{\beta_{1} \times 1}\right) \\
& =\sum_{m=0}^{\eta_{j}-1}\left(J_{i, w_{j}+m \beta_{2}}^{\beta_{1} \times 1}-J_{i, w_{j}+m \beta_{2}+\beta_{2}}^{\beta_{1} \times 1}\right) \\
& =\sum_{m=0}^{\eta_{j}-1}\left(J_{i, w_{j}+m \beta_{2}}^{\beta_{1} \times 1}-J_{i, w_{j}+(m+1) \beta_{2}}^{\beta_{1} \times 1}\right) \\
& =J_{i, w_{j}}^{\beta_{1} \times 1}-J_{i, w_{j}+\eta_{j} \beta_{2}}^{\beta_{1} \times 1} \\
& =J_{i, w_{j}}^{\beta_{1} \times 1}-J_{i, j}^{\beta_{1} \times 1} \\
& =C_{i, j}^{\beta_{1} \times 1} .
\end{aligned}
$$


Similarly, we can show that (2.10) is true.

To prove that $Q_{i, j}$ is a solution matrix, it is sufficient to show that $Q_{i, j}$ is a linear combination of $C_{i, j}^{\beta_{1} \times 1}$ which are solution matrices. Actually,

$$
\begin{aligned}
& \sum_{m=0}^{\zeta_{i}-1}\left(C_{u_{i}+m \beta_{1}, j}^{\beta_{1}}-C_{u_{i}+m \beta_{1}+1, j}^{\beta_{1} \times 1}\right) \\
= & \sum_{m=0}^{\zeta_{i}-1}\left[\left(J_{u_{i}+m \beta_{1}, w_{j}}^{\beta_{1} \times 1}-J_{u_{i}+m \beta_{1}, j}^{\beta_{1} \times 1}\right)-\left(J_{u_{i}+m \beta_{1}+1, w_{j}}^{\beta_{1} \times 1}-J_{u_{i}+m \beta_{1}+1, j}^{\beta_{1} \times 1}\right)\right] \\
= & \sum_{m=0}^{\zeta_{i}-1}\left[\left(J_{u_{i}+m \beta_{1}, w_{j}}^{\beta_{1} \times 1}-J_{u_{i}+m \beta_{1}+1, w_{j}}^{\beta_{1} \times 1}\right)+\left(-J_{u_{i}+m \beta_{1}, j}^{\beta_{1} \times 1}+J_{u_{i}+m \beta_{1}+1, j}^{\beta_{1} \times 1}\right)\right] \\
= & \sum_{m=0}^{\zeta_{i}-1}\left[\left(E_{u_{i}+m \beta_{1}, w_{j}}-E_{u_{i}+(m+1) \beta_{1}, w_{j}}\right)+\left(-E_{u_{i}+m \beta_{1}, j}+E_{u_{i}+(m+1) \beta_{1}, j}\right)\right] \\
= & \sum_{m=0}^{\zeta_{i}-1}\left(E_{u_{i}+m \beta_{1}, w_{j}}-E_{u_{i}+(m+1) \beta_{1}, w_{j}}\right)+\sum_{m=0}^{\zeta_{i}-1}\left(-E_{u_{i}+m \beta_{1}, j}+E_{u_{i}+(m+1) \beta_{1}, j}\right) \\
= & \left(E_{u_{i}, w_{j}}-E_{u_{i}+\zeta_{i} \beta_{i}, w_{j}}\right)+\left(-E_{u_{i}, j}+E_{u_{i}+\zeta_{i} \beta_{i}, j}\right) \\
= & E_{u_{i}, w_{j}}-E_{i, w_{j}}-E_{u_{i}, j}+E_{i, j} .
\end{aligned}
$$

The last equality holds by (2.8).

Now we define a function $\mathcal{T}: \mathcal{M}\left(\left(\alpha_{1} \times \alpha_{2}\right), n\right) \rightarrow \mathcal{M}\left(\left(\alpha_{1} \times \alpha_{2}\right), n\right)$ by

$$
\mathcal{T}(A)=A-\sum_{j=0}^{\alpha_{2}-1} \sum_{i=0}^{\alpha_{1}-1}[A]_{i, j} Q_{i, j} .
$$

By the definition, for $a, b, 0 \leq a \leq \alpha_{1}-1,0 \leq b \leq \alpha_{2}-1$,

$$
\mathcal{T}\left(E_{a, b}\right)=E_{u_{a}, b}+E_{a, w_{b}}-E_{u_{a}, w_{b}} .
$$

By (1.1), the following lemma immediately holds:

Lemma 2.2. The function $\mathcal{T}$ on $\mathcal{M}\left(\left(\alpha_{1}, \alpha_{2}\right), n\right)$ defined by (2.11) is a module homomorphism.

From Lemma 2.1, we know that $Q_{i, j}$ is a solution matrix of the $\left(\left(\alpha_{1}, \alpha_{2}\right) ;\left(\beta_{1}, \beta_{2}\right) ; n\right)$-DR game. Thus, the following lemma is true.

Lemma 2.3. Matrix $A \in \mathcal{M}\left(\left(\alpha_{1}, \alpha_{2}\right), n\right)$ is a solution matrix of the $\left(\left(\alpha_{1}, \alpha_{2}\right) ;\left(\beta_{1}, \beta_{2}\right) ; n\right)$ $D R$ game if and only if $\mathcal{T}(A)$ is a solution matrix of the $\left(\left(\alpha_{1}, \alpha_{2}\right) ;\left(\beta_{1}, \beta_{2}\right) ; n\right)$-DR game. 
We define a function $\mathcal{S}$ from $\mathcal{M}\left(\left(\alpha_{1}, \alpha_{2}\right), n\right)$ to $\mathcal{M}\left(\left(\alpha_{1}, \alpha_{2}\right), n\right)$ by

$$
\mathcal{S}(A)=\mathcal{T}(A)+\sum_{i=0}^{\alpha_{1}-1}[\mathcal{T}(A)]_{i, 0} R_{i, 0}^{1 \times g_{2}}+\sum_{j=0}^{\alpha_{2}-1}[\mathcal{T}(A)]_{0, j} C_{0, j}^{g_{1} \times 1}
$$

Then, by (1.1) and Lemma 2.2, the following lemma holds:

Lemma 2.4. The function $\mathcal{S}$ defined by (2.13) is a module homomorphism.

The following theorem is our main result which gives a characterization of the solution matrices.

Theorem 2.5. A matrix $A$ in $\mathcal{M}\left(\left(\alpha_{1}, \alpha_{2}\right), n\right)$ is a solution matrix of the $\left(\left(\alpha_{1}, \alpha_{2}\right) ;\left(\beta_{1}, \beta_{2}\right)\right.$; $n)$-DR game if and only if

$$
\mathcal{T}(A)=s_{1} \sum_{j=0}^{\alpha_{2}-1} d_{j} J_{0, j}^{g_{1} \times 1}+s_{2} \sum_{i=0}^{\alpha_{1}-1} e_{i} J_{i, 0}^{1 \times g_{2}}-s_{1} s_{2} t J_{0,0}^{g_{1} \times g_{2}}
$$

for some $d_{j}, e_{i}, t \in \mathbb{Z}_{n}$ and, specifically,

$$
\mathcal{S}(A)=s_{1} s_{2} t J_{0,0}^{g_{1} \times g_{2}}
$$

Theorem 2.5 tells us that we can determine whether or not a given matrix $A$ is a solution matrix by computing $\mathcal{T}(A)$ and $\mathcal{S}(A)$. Furthermore we have an $O\left(\alpha_{1} \alpha_{2}\right)$-algorithm to determine if a matrix is a solution matrix. By Theorem 2.5, we need to compute $\mathcal{T}$ and $\mathcal{S}$ given in (2.11) and check two equalities given in the theorem. The algorithm for computing the function $\mathcal{T}$ given in (2.11) iterates $\alpha_{1} \alpha_{2}$ times as each $Q_{i, j}$ has at most 4 nonzero components. Thus the time complexity for computing $\mathcal{T}$ is $O\left(\alpha_{1} \alpha_{2}\right)$. Similarly, the algorithm for computing the function $\mathcal{S}$ iterates $\alpha_{1}$ times for each $R_{i, 0}^{1 \times g_{2}}$ as it has at most $2 \alpha_{2}$ components, and iterates $\alpha_{2}$ times for each $C_{0, j}^{g_{1} \times 1}$ as it has at most $2 \alpha_{1}$ components. Therefore the complexity for computing $\mathcal{S}$ is $O\left(\alpha_{1} \alpha_{2}\right)$. Now it remains to check two equalities hold. This involves scanning the nonzero components and solving congruence equations in the form of $p q t=r(\bmod n)$ or $p q=r(\bmod n)$ where at least one of $p, q$ is $s_{1}$ or $s_{2}$. This also takes $O\left(\alpha_{1} \alpha_{2}\right)$. Hence the time complexity to determine if the matrix is a solution matrix is $O\left(\alpha_{1} \alpha_{2}\right)$.

In the rest of section, we devote ourselves to prove Theorem 2.5. To do so, we need several lemmas.

Lemma 2.6. For any integers $i$ and $j$, matrices $s_{2} C_{0, j}^{g_{1} \times 1}$ and $s_{1} R_{i, 0}^{1 \times g_{2}}$ are solution matrices of the $\left(\left(\alpha_{1}, \alpha_{2}\right) ;\left(\beta_{1}, \beta_{2}\right) ; n\right)-D R$ game. 
Proof. By (2.2), (2.12), and Lemma 2.2,

$$
\begin{aligned}
\mathcal{T}\left(C_{0, j}^{\beta_{1} \times 1}\right)= & \mathcal{T}\left(J_{0, w_{j}}^{\beta_{1} \times 1}-J_{0, j}^{\beta_{1} \times 1}\right)=\mathcal{T}\left(\sum_{a=0}^{\beta_{1}-1}\left(E_{a, w_{j}}-E_{a, j}\right)\right)=\sum_{a=0}^{\beta_{1}-1}\left(\mathcal{T}\left(E_{a, w_{j}}\right)-\mathcal{T}\left(E_{a, j}\right)\right) \\
= & \sum_{a=0}^{\beta_{1}-1}\left[\left(E_{u_{a}, w_{j}}+E_{a, w_{j}}-E_{u_{a}, w_{j}}\right)-\left(E_{u_{a}, j}+E_{a, w_{j}}-E_{u_{a}, w_{j}}\right)\right] \\
= & \sum_{a=0}^{\beta_{1}-1}\left(E_{u_{a}, w_{j}}-E_{u_{a}, j}\right)_{\text {by (2.1) }}^{=} s_{1} \sum_{a=0}^{g_{1}-1}\left(E_{a, w_{j}}-E_{a, j}\right)=s_{1}\left(J_{0, w_{j}}^{g_{1} \times 1}-J_{0, j}^{g_{1} \times 1}\right)=s_{1} C_{0, j}^{g_{1} \times 1} .
\end{aligned}
$$

Similarly, we can show that

$$
\mathcal{T}\left(R_{i, 0}^{1 \times \beta_{2}}\right)=s_{2} R_{i, 0}^{1 \times g_{2}}
$$

As $\mathcal{T}\left(C_{0, j}^{\beta_{1} \times 1}\right)$ and $\mathcal{T}\left(R_{i, 0}^{1 \times \beta_{2}}\right)$ are solution matrices by Lemmas 2.1 and 2.3 , the lemma follows.

Lemma 2.7. For any two integers $i, j$,

$$
\mathcal{T}\left(J_{i, j}^{*}\right)=s_{1} J_{0, j}^{g_{1} \times \beta_{2}}+s_{2} J_{i, 0}^{\beta_{1} \times g_{2}}-s_{1} s_{2} J_{0,0}^{g_{1} \times g_{2}} .
$$

Proof. By Lemma 2.2,

$$
\begin{aligned}
\mathcal{T}\left(J_{i, j}^{*}\right) & =\mathcal{T}\left(\sum_{b=j}^{j+\beta_{2}-1} \sum_{a=i}^{i+\beta_{1}-1} E_{a, b}\right)=\sum_{b=j}^{j+\beta_{2}-1} \sum_{a=i}^{i+\beta_{1}-1} \mathcal{T}\left(E_{a, b}\right) \\
& =\sum_{b=j}^{j+\beta_{2}-1} \sum_{a=i}^{i+\beta_{1}-1}\left(E_{u_{a}, b}+E_{a, w_{b}}-E_{u_{a}, w_{b}}\right) \\
& =\sum_{b=j}^{j+\beta_{2}-1} \sum_{a=i}^{i+\beta_{1}-1} E_{u_{a}, b}+\sum_{b=j}^{j+\beta_{2}-1} \sum_{a=i}^{i+\beta_{1}-1} E_{a, w_{b}}-\sum_{b=j}^{j+\beta_{2}-1} \sum_{a=i}^{i+\beta_{1}-1} E_{u_{a}, w_{b}} \\
& =\sum_{b=j}^{j+\beta_{2}-1}\left(s_{1} \sum_{a=0}^{g_{1}-1} E_{a, b}\right)+\sum_{a=i}^{i+\beta_{1}-1}\left(s_{2} \sum_{b=0}^{g_{2}-1} E_{a, b}\right)-s_{1} s_{2} \sum_{b=0}^{g_{2}-1} \sum_{a=0}^{g_{1}-1} E_{a, b} \\
& =s_{1} \sum_{b=j}^{j+\beta_{2}-1} \sum_{a=0}^{g_{1}-1} E_{a, b}+s_{2} \sum_{a=i}^{i+\beta_{1}-1} \sum_{b=0}^{g_{2}-1} E_{a, b}-s_{1} s_{2} \sum_{b=0}^{g_{2}-1} \sum_{a=0}^{g_{1}-1} E_{a, b} \\
& =s_{1} J_{0, j}^{g_{1} \times \beta_{2}}+s_{2} J_{i, 0}^{\beta_{1} \times g_{2}}-s_{1} s_{2} J_{0,0}^{g_{1} \times g_{2}} .
\end{aligned}
$$

Therefore, the lemma holds.

Lemma 2.8. For any integers $i, j, \mathcal{S}\left(J_{i, j}^{*}\right)$ is a solution matrix of the $\left(\left(\alpha_{1}, \alpha_{2}\right) ;\left(\beta_{1}, \beta_{2}\right) ; n\right)$ DR game and

$$
\mathcal{S}\left(J_{i, j}^{*}\right)=s_{1} s_{2} J_{0,0}^{g_{1} \times g_{2}}
$$


Proof. First, we will show that $\left.\sum_{a=0}^{\alpha_{1}-1}\left[\mathcal{T}\left(J_{i, j}^{*}\right)\right)\right]_{a, 0} R_{a, 0}^{1 \times g_{2}}$ is a solution matrix and that

$$
\left.\sum_{a=0}^{\alpha_{1}-1}\left[\mathcal{T}\left(J_{i, j}^{*}\right)\right)\right]_{a, 0} R_{a, 0}^{1 \times g_{2}}=s_{2}\left(s_{1} J_{0,0}^{g_{1} \times g_{2}}-J_{i, 0}^{\beta_{1} \times g_{2}}\right) .
$$

Consider the case where $\alpha_{1}=\beta_{1}$. Then $g_{1}=\operatorname{gcd}\left(\alpha_{1}, \beta_{1}\right)=\alpha_{1}$ and $s_{1}=1$. Then by (2.6), it holds that

$$
\sum_{a=0}^{\alpha_{1}-1}\left[\mathcal{T}\left(J_{i, j}^{*}\right)\right]_{a, 0} R_{a, 0}^{1 \times g_{2}}=O
$$

and so $\left.\sum_{a=0}^{\alpha_{1}-1}\left[\mathcal{T}\left(J_{i, j}^{*}\right)\right)\right]_{a, 0} R_{a, 0}^{1 \times g_{2}}$ is a trivial solution matrix. On the other hand, since $s_{1}=1$ and $g_{1}=\beta_{1}$,

$$
s_{2}\left(s_{1} J_{0,0}^{g_{1} \times g_{2}}-J_{i, 0}^{\beta_{1} \times g_{2}}\right)=s_{2}\left(J_{0,0}^{g_{1} \times g_{2}}-J_{0,0}^{g_{1} \times g_{2}}\right)
$$

and so the equality (2.14) holds.

Now consider the case where $\alpha_{1}>\beta_{1}$. Then $g_{1}<\alpha_{1}$. Let $X=\left\{0,1,2, \ldots, g_{1}-1\right\}$, $Y=\left\{g_{1}, \ldots, \alpha_{1}-1\right\}$, and $Z=\left\{i, i+1 \ldots, i+\beta_{1}-1\right\}$. By Lemma 2.7, for $a, 0 \leq a \leq \alpha_{1}-1$,

$$
\left[\mathcal{T}\left(J_{i, j}^{*}\right)\right]_{a, 0}=s_{1}\left[J_{0, j}^{g_{1} \times \beta_{2}}\right]_{a, 0}+s_{2}\left[J_{i, 0}^{\beta_{1} \times g_{2}}\right]_{a, 0}-s_{1} s_{2}\left[J_{0,0}^{g_{1} \times g_{2}}\right]_{a, 0} .
$$

For $a \in Y, s_{1}\left[J_{0, j}^{g_{1} \times \beta_{2}}\right]_{a, 0}=0$ and $\left[J_{0,0}^{g_{1} \times g_{2}}\right]_{a, 0}=0$. Thus

$$
\left[\mathcal{T}\left(J_{i, j}^{*}\right)\right]_{a, 0}= \begin{cases}s_{2} & \text { if } a \in Y \cap Z \\ 0 & \text { if } Y \backslash Z\end{cases}
$$

Then

$$
\begin{aligned}
\sum_{a \in X}\left[\mathcal{T}\left(J_{i, j}^{*}\right)\right]_{a, 0} R_{a, 0}^{1 \times g_{2}} & \underset{\text { by }}{=} \underset{\text { (2.6) }}{=} \sum_{a \in Y}\left[\mathcal{T}\left(J_{i, j}^{*}\right)\right]_{a, 0} R_{a, 0}^{1 \times g_{2}} \underset{\text { by }}{=}=\sum_{\substack{(2.15) \\
=}} \sum_{a \in Y \cap Z} s_{2} R_{a, 0}^{1 \times g_{2}} \\
& \sum_{a \in Y \cap Z} s_{2} R_{a, 0}^{1 \times g_{2}}+\sum_{a \in X \cap Z} s_{2} R_{a, 0}^{1 \times g_{2}}=\sum_{a \in Z} s_{2} R_{a, 0}^{1 \times g_{2}} .
\end{aligned}
$$

Therefore,

$$
\sum_{a \in X}\left[\mathcal{T}\left(J_{i, j}^{*}\right)\right]_{a, 0} R_{a, 0}^{1 \times g_{2}}=\sum_{a=0}^{\alpha_{1}-1}\left[\mathcal{T}\left(J_{i, j}^{*}\right)\right]_{a, 0} R_{a, 0}^{1 \times g_{2}}=\sum_{a \in Z} s_{2} R_{a, 0}^{1 \times g_{2}}=\sum_{a=i}^{i+\beta_{1}-1} s_{2} R_{a, 0}^{1 \times g_{2}}
$$


and so $\left.\sum_{a=0}^{\alpha_{1}-1}\left[\mathcal{T}\left(J_{i, j}^{*}\right)\right)\right]_{a, 0} R_{a, 0}^{1 \times g_{2}}$ is a solution matrix by Lemma 2.6. In addition,

$$
\begin{aligned}
\sum_{a=i}^{i+\beta_{1}-1} s_{2} R_{a, 0}^{1 \times g_{2}} & =s_{2} \sum_{a=i}^{i+\beta_{1}-1}\left(J_{u_{a}, 0}^{1 \times g_{2}}-J_{a, 0}^{1 \times g_{2}}\right)=s_{2} \sum_{a=i}^{i+\beta_{1}-1}\left(\sum_{b=0}^{g_{2}-1}\left(E_{u_{a}, b}-E_{a, b}\right)\right) \\
& =s_{2} \sum_{b=0}^{g_{2}-1} \sum_{a=i}^{i+\beta_{1}-1}\left(E_{u_{a}, b}-E_{a, b}\right)=s_{2}\left(\sum_{b=0}^{g_{2}-1} \sum_{a=i}^{i+\beta_{1}-1} E_{u_{a}, b}-\sum_{b=0}^{g_{2}-1} \sum_{a=i}^{i+\beta_{1}-1} E_{a, b}\right) \\
& =s_{2}\left(s_{1} \sum_{b=0}^{g_{2}-1} \sum_{a=0}^{g_{1}-1} E_{a, b}-J_{i, 0}^{\beta_{1} \times g_{2}}\right)=s_{2}\left(s_{1} J_{0,0}^{g_{1} \times g_{2}}-J_{i, 0}^{\beta_{1} \times g_{2}}\right) .
\end{aligned}
$$

Therefore, (2.14) holds. Similarly, we can show that $\sum_{b=0}^{\alpha_{2}-1}\left[\mathcal{T}\left(J_{i, j}^{*}\right)\right]_{0, b} C_{0, b}^{g_{1} \times 1}$ is a solution matrix and that

$$
\sum_{b=0}^{\alpha_{2}-1}\left[\mathcal{T}\left(J_{i, j}^{*}\right)\right]_{0, b} C_{0, b}^{g_{1} \times 1}=s_{1}\left(s_{2} J_{0,0}^{g_{1} \times g_{2}}-J_{0, j}^{g_{1} \times \beta_{2}}\right) .
$$

By the definition of $\mathcal{S}$ given in (2.13),

$$
\mathcal{S}\left(J_{i, j}^{*}\right)=\mathcal{T}\left(J_{i, j}^{*}\right)+\sum_{a=0}^{\alpha_{1}-1}\left[\mathcal{T}\left(J_{i, j}^{*}\right)\right]_{a, 0} R_{a, 0}^{1 \times g_{2}}+\sum_{b=0}^{\alpha_{2}-1}\left[\mathcal{T}\left(J_{i, j}^{*}\right)\right]_{0, b} C_{0, b}^{g_{1} \times 1} .
$$

Note that both $\sum_{a=0}^{\alpha_{1}-1}\left[\mathcal{T}\left(J_{i, j}^{*}\right)\right]_{a, 0} R_{a, 0}^{1 \times g_{2}}$ and $\sum_{b=0}^{\alpha_{2}-1}\left[\mathcal{T}\left(J_{i, j}^{*}\right)\right]_{0, b} C_{0, b}^{g_{1} \times 1}$ are solution matrices. Furthermore, by Lemma $2.3, \mathcal{T}\left(J_{i, j}^{*}\right)$ is a solution matrix. Therefore, $\mathcal{S}\left(J_{i, j}^{*}\right)$ is a solution matrix. On the other hand, from (2.14), (2.16) and Lemma 2.7, it follows that

$$
\begin{aligned}
\mathcal{S}\left(J_{i, j}^{*}\right) & =s_{1} J_{0, j}^{g_{1} \times \beta_{2}}+s_{2} J_{i, 0}^{\beta_{1} \times g_{2}}-s_{1} s_{2} J_{0,0}^{g_{1} \times g_{2}}+s_{2}\left(s_{1} J_{0,0}^{g_{1} \times g_{2}}-J_{i, 0}^{\beta_{1} \times g_{2}}\right)+s_{1}\left(s_{2} J_{0,0}^{g_{1} \times g_{2}}-J_{0, j}^{g_{1} \times \beta_{2}}\right) \\
& =s_{1} s_{2} J_{0,0}^{g_{1} \times g_{2}} .
\end{aligned}
$$

Hence the lemma holds.

Now we are ready to prove the main result:

Proof of Theorem 2.5. Suppose that a matrix $A$ in $\mathcal{M}\left(\left(\alpha_{1}, \alpha_{2}\right), n\right)$ is a solution matrix of the $\left(\left(\alpha_{1}, \alpha_{2}\right) ;\left(\beta_{1}, \beta_{2}\right) ; n\right)$-DR game. By definition, there exists a solving coefficient matrix $\left(c_{i, j}\right)$ of the $\left(\left(\alpha_{1}, \alpha_{2}\right) ;\left(\beta_{1}, \beta_{2}\right) ; n\right)$-DR game corresponding to $A$. That is,

$$
A=-\sum_{b=0}^{\alpha_{2}-1} \sum_{a=0}^{\alpha_{1}-1} c_{a, b} J_{a, b}^{*}
$$


Then

$$
\begin{aligned}
\mathcal{T}(A)= & \sum_{b=0}^{\alpha_{2}-1} \sum_{a=0}^{\alpha_{1}-1}\left(-c_{a, b}\right) \mathcal{T}\left(J_{a, b}^{*}\right) \\
= & \sum_{b=0}^{\alpha_{2}-1} \sum_{a=0}^{\alpha_{1}-1}\left(-c_{a, b}\right)\left(s_{1} J_{0, b}^{g_{1} \times \beta_{2}}+s_{2} J_{a, 0}^{\beta_{1} \times g_{2}}-s_{1} s_{2} J_{0,0}^{g_{1} \times g_{2}}\right) \quad \text { (by Le Le } \\
= & \sum_{b=0}^{\alpha_{2}-1} \sum_{a=0}^{\alpha_{1}-1}\left(-c_{a, b}\right)\left(s_{1} \sum_{j=b}^{b+\beta_{2}-1} J_{0, j}^{g_{1} \times 1}+s_{2} \sum_{i=a}^{a+\beta_{1}-1} J_{i, 0}^{1 \times g_{2}}-s_{1} s_{2} J_{0,0}^{g_{1} \times g_{2}}\right) \\
= & s_{1} \sum_{b=0}^{\alpha_{2}-1} \sum_{j=b}^{b+\beta_{2}-1} \sum_{a=0}^{\alpha_{1}-1}\left(-c_{a, b}\right) J_{0, j}^{g_{1} \times 1}+s_{2} \sum_{a=0}^{\alpha_{1}-1} \sum_{i=a}^{a+\beta_{1}-1} \sum_{b=0}^{\alpha_{2}-1}\left(-c_{a, b}\right) J_{i, 0}^{1 \times g_{2}} \\
& -s_{1} s_{2} \sum_{b=0}^{\alpha_{2}-1} \sum_{a=0}^{\alpha_{1}-1}\left(-c_{a, b}\right) J_{0,0}^{g_{1} \times g_{2}} .
\end{aligned}
$$

It is not difficult to check that

$$
\begin{aligned}
& s_{1} \sum_{b=0}^{\alpha_{2}-1} \sum_{j=b}^{b+\beta_{2}-1} \sum_{a=0}^{\alpha_{1}-1}\left(-c_{a, b}\right) J_{0, j}^{g_{1} \times 1}=s_{1} \sum_{j=0}^{\alpha_{2}-1} \sum_{b=j-\beta_{2}+1}^{j} \sum_{a=0}^{\alpha_{1}-1}\left(-c_{a, b}\right) J_{0, j}^{g_{1} \times 1}=s_{1} \sum_{j=0}^{\alpha_{2}-1} d_{j} J_{0, j}^{g_{1} \times 1} \\
& s_{2} \sum_{a=0}^{\alpha_{1}-1} \sum_{i=a}^{a+\beta_{1}-1} \sum_{b=0}^{\alpha_{2}-1}\left(-c_{a, b}\right) J_{i, 0}^{1 \times g_{2}}=s_{2} \sum_{i=0}^{\alpha_{1}-1} \sum_{a=i-\beta_{1}+1}^{i} \sum_{b=0}^{\alpha_{2}-1}\left(-c_{a, b}\right) J_{i, 0}^{1 \times g_{2}}=s_{2} \sum_{a=0}^{\alpha_{1}-1} e_{i} J_{i, 0}^{1 \times g_{2}},
\end{aligned}
$$

where $d_{j}=\sum_{b=j-\beta_{2}+1}^{j} \sum_{a=0}^{\alpha_{1}-1}\left(-c_{a, b}\right)$ and $e_{i}=\sum_{a=i-\beta_{1}+1}^{i} \sum_{b=0}^{\alpha_{2}-1}\left(-c_{a, b}\right)$. Thus we obtain

$$
\mathcal{T}(A)=s_{1} \sum_{j=0}^{\alpha_{2}-1} d_{j} J_{0, j}^{g_{1} \times 1}+s_{2} \sum_{a=0}^{\alpha_{1}-1} e_{i} J_{i, 0}^{1 \times g_{2}}-s_{1} s_{2} t J_{0,0}^{g_{1} \times g_{2}}
$$

where $t=\sum_{b=0}^{\alpha_{2}-1} \sum_{a=0}^{\alpha_{1}-1}\left(-c_{a, b}\right)$. On the other hand, by Lemmas 2.4 and 2.8,

$$
\begin{aligned}
\mathcal{S}(A) & =\sum_{j=0}^{\alpha_{2}-1} \sum_{i=0}^{\alpha_{1}-1}\left(-c_{i, j}\right) \mathcal{S}\left(J_{i, j}^{*}\right)=\sum_{j=0}^{\alpha_{2}-1} \sum_{i=0}^{\alpha_{1}-1}\left(-c_{i, j}\right) s_{1} s_{2} J_{0,0}^{g_{1} \times g_{2}} \\
& =\left[\sum_{j=0}^{\alpha_{2}-1} \sum_{i=0}^{\alpha_{1}-1}\left(-c_{i, j}\right)\right] s_{1} s_{2} J_{0,0}^{g_{1} \times g_{2}}=s_{1} s_{2} t J_{0,0}^{g_{1} \times g_{2}} .
\end{aligned}
$$

Thus the 'only if' part is true. To show the converse, assume that

$$
\mathcal{T}(A)=s_{1} \sum_{j=0}^{\alpha_{2}-1} d_{j} J_{0, j}^{g_{1} \times 1}+s_{2} \sum_{i=0}^{\alpha_{1}-1} e_{i} J_{i, 0}^{1 \times g_{2}}-s_{1} s_{2} t J_{0,0}^{g_{1} \times g_{2}}
$$


and $\mathcal{S}(A)=s_{1} s_{2} t J_{0,0}^{g_{1} \times g_{2}}$ for some $d_{j}, e_{i}, t \in \mathbb{Z}_{n}$. Then, by the definition of $\mathcal{S}$ given in $(2.13)$

$$
s_{1} s_{2} t J_{0,0}^{g_{1} \times g_{2}}=\mathcal{T}(A)+\sum_{i=0}^{\alpha_{1}-1}[\mathcal{T}(A)]_{i, 0} R_{i, 0}^{1 \times g_{2}}+\sum_{j=0}^{\alpha_{2}-1}[\mathcal{T}(A)]_{0, j} C_{0, j}^{g_{1} \times 1},
$$

or

$$
\mathcal{T}(A)=s_{1} s_{2} t J_{0,0}^{g_{1} \times g_{2}}-\sum_{i=0}^{\alpha_{1}-1}[\mathcal{T}(A)]_{i, 0} R_{i, 0}^{1 \times g_{2}}-\sum_{j=0}^{\alpha_{2}-1}[\mathcal{T}(A)]_{0, j} C_{0, j}^{g_{1} \times 1} .
$$

The first term $s_{1} s_{2} t J_{0,0}^{g_{1} \times g_{2}}$ of the right hand side of above inequality is equal to $t \mathcal{S}\left(J_{i, j}^{*}\right)$ for some integers $i, j$ and so it is a solution matrix of the $\left(\left(\alpha_{1}, \alpha_{2}\right) ;\left(\beta_{1}, \beta_{2}\right) ; n\right)$-DR game by Lemma 2.8. By (2.5), (2.6) and (2.17), the matrix $[\mathcal{T}(A)]_{i, 0} R_{i, 0}^{1 \times g_{2}}$ is equal to either $O$ or $s_{2} e_{i} R_{i, 0}^{1 \times g_{2}}$, and the matrix $[\mathcal{T}(A)]_{0, j} C_{0, j}^{g_{1} \times 1}$ is equal to either $O$ or $s_{1} d_{j} C_{0, j}^{g_{1} \times 1}$, depending on whether or not $0 \leq i \leq g_{1}-1,0 \leq j \leq g_{2}-1$. Therefore, by Lemma 2.6, both $[\mathcal{T}(A)]_{i, 0} R_{i, 0}^{1 \times g_{2}}$ and $[\mathcal{T}(A)]_{0, j} C_{0, j}^{g_{1} \times 1}$ are solution matrices. Thus we can conclude that $\mathcal{T}(A)$ is a solution matrix. By Lemma $2.3, A$ is a solution matrix of the $\left(\left(\alpha_{1}, \alpha_{2}\right) ;\left(\beta_{1}, \beta_{2}\right) ; n\right)$-DR game.

\section{Closing remarks}

In this paper, we give a necessary and sufficient condition for a torus to be a solution of the $\left(\left(\alpha_{1}, \alpha_{2}\right) ;\left(\beta_{1}, \beta_{2}\right) ; n\right)$-DR game for positive integers $\alpha_{1}, \alpha_{2}, n, \beta_{1}, \beta_{2}$ such that $\beta_{1} \leq \alpha_{1}$ and $\beta_{2} \leq \alpha_{2}$.

When $\alpha_{1}=\beta_{1}=1$, a matrix in $\mathcal{M}\left(\left(\alpha_{1}, \alpha_{2}\right), n\right)$ becomes an $\alpha_{2}$-tuple, so $\mathcal{D}\left(\left(1, \alpha_{2}\right), n\right)$ is the set of circles on each of which $\alpha_{2} n$-dice are located. By using the results obtained in the previous section, we can characterize a circle which is a solution of dice rolling game on the set $\mathcal{D}\left(\left(1, \alpha_{2}\right), n\right)$ with respect to $\left(1, \beta_{2}\right)$-rolling procedures.

Suppose that $\alpha_{1}=\beta_{1}$. Then $g_{1}=\operatorname{gcd}\left(\alpha_{1}, \beta_{1}\right)=\alpha_{1}$ and so $s_{1}=1$. Then $0 \leq i \leq \alpha_{1}-1$ if an only if $0 \leq i \leq g_{1}-1$. By (2.7) and the definition of $\mathcal{T}$ given in (2.11), if $0 \leq i \leq g_{1}-1$ or $0 \leq j \leq g_{2}-1$, then $\mathcal{T}$ is the identity function, that is, $\mathcal{T}(A)=A$. Since $\alpha_{1}=g_{1}$, $R_{i, 0}^{1 \times g_{2}}=O$ by $(2.6)$ and so

$$
\mathcal{S}(A)=A+\sum_{j=0}^{\alpha_{2}-1}[A]_{0, j} C_{0, j}^{\alpha_{1} \times 1} .
$$

Hence a characterization of a solution matrix in $\mathcal{M}\left(\left(1, \alpha_{2}\right), n\right)$ immediately follows from Theorem 2.5:

$A$ matrix $A$ is a solution matrix of the $\left(\left(\alpha_{1}, \alpha_{2}\right) ;\left(\alpha_{1}, \beta_{2}\right) ; n\right)-D R$ game if and only if

$$
A=\sum_{j=0}^{\alpha_{2}-1} d_{j} J_{0, j}^{\alpha_{1} \times 1}+s_{2} \sum_{i=0}^{\alpha_{1}-1} e_{i} J_{i, 0}^{1 \times g_{2}}-s_{2} t J_{0,0}^{\alpha_{1} \times g_{2}}
$$

for some $d_{j}, e_{i}, t \in \mathbb{Z}_{n}$ and $\mathcal{S}(A)=u s_{2} J_{0,0}^{\alpha_{1} \times g_{2}}$ for some $u \in \mathbb{Z}_{n}$. 
However, if $\mathcal{S}(A)=u s_{2} J_{0,0}^{\alpha_{1} \times g_{2}}$ for some $u \in \mathbb{Z}_{n}$, then it holds that

$$
\begin{aligned}
A & =-\mathcal{S}(A)+\sum_{j=0}^{\alpha_{2}-1}[A]_{0, j} C_{0, j}^{\alpha_{1} \times 1} \\
& =-u s_{2} J_{0,0}^{\alpha_{1} \times g_{2}}+\sum_{j=0}^{\alpha_{2}-1}[A]_{0, j} C_{0, j}^{\alpha_{1} \times 1} \\
& =-u s_{2} \sum_{j=0}^{g_{2}-1} J_{0, j}^{\alpha_{1} \times 1}+\sum_{j=0}^{\alpha_{2}-1}[A]_{0, j}\left(J_{0, w_{j}}^{g_{1} \times 1}-J_{0, j}^{\alpha_{1} \times 1}\right) \\
& =\sum_{j=0}^{g_{2}-1}\left(-u s_{2}\right) J_{0, j}^{\alpha_{1} \times 1}+\sum_{j=0}^{\alpha_{2}-1}\left([A]_{0, j} J_{0, w_{j}}^{\alpha_{1} \times 1}-[A]_{0, j} J_{0, j}^{\alpha_{1} \times 1}\right) \\
& =\sum_{j=0}^{\alpha_{2}-1} f_{j} J_{0, j}^{\alpha_{1} \times 1}=s_{2} \sum_{i=0}^{\alpha_{1}-1} 0 \cdot J_{i, 0}^{1 \times g_{2}}+\sum_{j=0}^{\alpha_{2}-1} f_{j} J_{0, j}^{\alpha_{1} \times 1}-s_{2} \cdot 0 \cdot J_{0,0}^{\alpha_{1} \times g_{2}}
\end{aligned}
$$

for some $f_{j} \in \mathbb{Z}_{n}$. Thus (3.2) is true if $\mathcal{S}(A)=u s_{2} J_{0,0}^{\alpha_{1} \times g_{2}}$ for some $u \in \mathbb{Z}_{n}$. Hence the characterization of a solution matrix in $\mathcal{M}\left(\left(1, \alpha_{2}\right), n\right)$ given above can be simplified as follows:

Corollary 3.1. A matrix $A$ is a solution matrix of the $\left(\left(\alpha_{1}, \alpha_{2}\right) ;\left(\alpha_{1}, \beta_{2}\right) ; n\right)-D R$ game if and only if

$$
\mathcal{S}(A)=u s_{2} J_{0,0}^{\alpha_{1} \times g_{2}}
$$

for some $u \in \mathbb{Z}_{n}$.

When $\alpha_{1}=\beta_{1}=1$, the above proposition can be stated as:

An ordered $\alpha_{2}$-tuple $\mathbf{v}$ is a solution matrix of the $\left(\left(1, \alpha_{2}\right) ;\left(1, \beta_{2}\right) ; n\right)$-DR game if and only if $\mathcal{S}(\mathbf{v})=(\underbrace{u s_{2}, \ldots, u s_{2}}_{g_{2}}, 0, \ldots, 0)$ for some $u \in \mathbb{Z}_{n}$.

which characterizes the solution set of the dice rolling game on a set of circles.

For the case where $\alpha_{2}=\beta_{2}$, we can give a similar argument. We believe that our characterization for the 2 -dimensional case can be generalized to the $t$-dimensional case for $t \geq 1$ if we can find a way to manipulate notations more effectively.

Acknowledgments. We wish to acknowledge an anonymous referee for suggestions to improve the introduction part of this paper. 


\section{References}

[1] R. Barura, S. Ramakrishnan: $\sigma^{-}$-game, $\sigma^{+}$-game and two-dimensional additive cellunar automata, Theor. Comput. Sci. 154 (1996), 249-366.

[2] M. Florence, F. Meunier: Completely symmetric configurations for $\sigma$-games on grid graphs, J. Algebr. Comb. 31 (2010), 533-545.

[3] J. Goldwasser, X. Wu: Does the lit-only restriction make any defference for the $\sigma$-game and $\sigma^{+}$-game? Eur. J. Comb. 30 (2009), 774-787.

[4] M. Hunziker, A. Machiavelo, J. Park: Chebyshev polynomials over finite fiels and reversibility of $\sigma$-automata on square grids, Theoret. Comput. Sci. 320 (2004), 465483.

[5] S-G. Lee, J-M. Yang: Linear algebraic approach on real $\sigma$-game: J. Appl. Math. \& Computing 21 (2006), 295-305.

[6] K. Sutner: Linear cellular automata and the Garden-of-Eden. Math. Intell. 11 (1989), $49-53$.

[7] K. Sutner: $\sigma$-automata and Chebychev-polynomials. Theor. Comput. Sci. 230 (2000), 24-34. 